\title{
VALIDACIÓN Y APLICACIÓN DE UNA ESCALA DE CONOCIMIENTOS Y PRÁCTICAS SOBRE HIGIENIZACIÓN DE MANOS EN ESTUDIANTES DE ODONTOLOGÍA
}

Recibido: octubre del 2018

Aceptado: junio del 2019

John Querubín Franco-Aguirre ${ }^{1}$, Ángela María Sarrazola-Moncada ${ }^{2}$

\section{Resumen}

Objetivo: Validar y aplicar una escala de conocimientos y prácticas en higienización de las manos en estudiantes de odontología. Metodología: Estudio longitudinal de validación y aplicación de una escala e intervención educativa en una población de 39 estudiantes de odontología. Se empleó una fuente de información primaria que consiste en una escala de conocimientos y prácticas; la fiabilidad se evaluó con el coeficiente de alfa de Cronbach; la consistencia interna y la validez discriminante con coeficientes de correlación de Pearson y la validez de contenido y constructo mediante el análisis factorial exploratorio de componentes principales. La efectividad de la intervención educativa respecto a los conocimientos y prácticas se evalúo con un análisis bivariado. Resultados: El porcentaje de éxito en la consistencia interna fue de 77,2\% para los ítems de conocimientos y 72,7\% para los de prácticas; la validez discriminante fue del $100 \%$ para ambos dominios; para la validez de contenido y constructo, el porcentaje de éxito fue del $100 \%$; en la fiabilidad se obtuvieron alfa de Cronbach iguales a 0,7. Los puntajes en los dominios de conocimientos y prácticas fueron significativamente mejores después de la intervención educativa. Conclusiones: Se halló una excelente confiabilidad y validez en la escala construida, lo que representa la disponibilidad de un instrumento para la evaluación de los conocimientos y prácticas en higienización de las manos en profesionales de la salud y la orientación de procesos de información, comunicación y educación.

Palabras clave: conocimientos, higienización de manos, infecciones relacionadas a la asistencia sanitaria, intervención educativa, prácticas, psicometría.

1 Facultad de Odontología-Universidad Cooperativa de Colombia y Escuela de Microbiología-Universidad de Antioquia. Dirección de correspondencia: Carrera 47 No. 37 Sur - 18 Envigado -Antioquia. Teléfono: 4446065 Ext. 4111 Código postal: 055422. Correo electrónico: john.francoa@campusucc.edu.co

${ }^{2}$ Facultad de Odontología-Universidad Cooperativa de Colombia. 


\section{VALIDATION AND APPLICATION OF A SCALE OF KNOWLEDGE AND PRACTICES ABOUT HAND SANITATION IN DENTISTRY STUDENTS}

John Querubín Franco-Aguirre ${ }^{1}$, Ángela María Sarrazola-Moncada ${ }^{2}$

\section{Abstract}

Objective: To validate and apply a scale of knowledge and practices in hand sanitation in dentistry students. Method: Longitudinal study to validate and apply a scale of educational intervention in a population of 39 dentistry students. We used a primary source of information which consists of the scale; reliability was evaluated with Cronbach's coefficient; internal consistency and validity were evaluated with chi-squared coefficients and content validity was evaluated through exploratory analysis of main components. Effectiveness of the pedagogical intervention in terms of knowledge and practices was evaluated with a two-variable analysis. Results: The percentage of success in internal consistency was $77.2 \%$ for knowledge items and $72.7 \%$ for the practicum items. Validity was of $100 \%$ for both, and content had a success percentage of $100 \%$ as well. Reliability was 0.7 . The scores in knowledge and practices were quite better after the pedagogical intervention. Conclusion: We found excellent reliability and validity in the built scale, which represents the availability of an instrument for evaluation of knowledge and practice in hand sanitization in professionals of the health areas and the orientation in the process of information, communication and education. 


\section{VALIDAÇÃO E APLICAÇÃOO DE UMA ESCALA DE CONHECIMENTOS E PRÁTICAS SOBRE HIGIENIZAÇÃO DE MÃOS EM ESTUDANTES DE ODONTOLOGIA}

John Querubín Franco-Aguirre' ${ }^{1}$ Ángela María Sarrazola-Moncada²

\section{Resumo}

Objetivo: validar e aplicar uma escala de conhecimentos e práticas em higienização das mãos em estudantes de Odontologia. Metodologia: estudolongitudinaldevalidação e aplicação de uma escalae intervenção educativa com uma população de 39 estudantes de Odontologia. Foi utilizada uma fonte de informação primária que consiste em uma escala de conhecimentos e práticas; a confiabilidade foi avaliada com o coeficiente de alfa de Cronbach; a consistência interna e a validade discriminante com coeficientes de correlação de Pearson, e a validade de conteúdo e constructo mediante a análise fatorial exploratória de componentes principais. A efetividade da intervenção educativa quanto aos conhecimentos e às práticas foi avaliada com uma análise bivariada. Resultados: a porcentagem de sucesso na consistência interna foi de $77,2 \%$ para os itens de conhecimentos e $72,7 \%$ para os de práticas; a validade discriminante foi de $100 \%$ para ambos os domínios; para a validade de conteúdo e constructo, a porcentagem de sucesso foi de $100 \%$; na confiabilidade, foram obtidos alfas de Cronbach iguais a 0,7. As pontuações nos mesmos domínios de conhecimento e práticas foram significativamente melhores depois da intervenção educativa. Conclusões: foi verificada excelente confiabilidade e validade na escala construída, o que representa a disponibilidade de um instrumento para avaliar os conhecimentos e práticas em higienização das mãos em profissionais da saúde e a orientação de processos de informação, comunicação e educação. 


\section{Introducción}

Las infecciones relacionadas con la asistencia sanitaria (IRAS) son aquellas que el paciente puede contraer al recibir atención en salud o durante su permanencia en un centro asistencial. El CDC (Center for Disease Control) de Atlanta las ha definido como: "todo cuadro clínico, localizado o sistémico, como resultado de una reacción adversa dada la presencia de uno o varios agentes infecciosos o sus toxinas, sin evidencia de que estuviese presente o en fase de incubación al momento del ingreso hospitalario" $(1,2)$.

Entre los servicios de asistencia sanitaria identificados se encuentra el ambulatorio, tipificado como todo servicio asistencial prestado a pacientes que no son ingresados en un hospital de cuidados no agudos, de los cuales se incluyen los servicios de consulta odontológica como uno de los ámbitos de exposición para la adquisición de IRAS, sin embargo, este al igual que otros tipos de servicios ambulatorios han sido considerados entornos de bajo riesgo, debido en parte a la exigua información sobre su ocurrencia, producto de la dificultad de su diagnóstico por la corta estancia del paciente y la imposibilidad de diferenciarlas de las infecciones comunitarias $(1,2)$.

A pesar de lo anterior, algunos estudios han logrado evidenciar la magnitud de las IRAS como un fenómeno presente en los servicios de atención ambulatoria, como es el caso de la investigación

realizada por Aranaz, et al., en 48 centros de atención primaria en España sobre la identificación de eventos adversos derivados de procedimientos de atención ambulatoria, en los cuales se describieron 57 casos de infecciones relacionadas con los cuidados de salud en un total de 96047 consultas, de dichos casos el 78,9\% se consideraron evitables (3); otro estudio prospectivo longitudinal realizado por Schulz, Mielke \& Wischnewski en centros geriátricos de Alemania, describió una incidencia de 393 infecciones en 243 pacientes de un total de 511, durante 10 meses de seguimiento entre 2008 y 2009 (4).

Como vector de trasmisión de agentes infecciosos a los pacientes durante la atención ambulatoria, se ha evidenciado que las manos del personal asistencial representan una fuente potencial de transferencia, como lo demostraron Aguilar y colaboradores (5) en un estudio realizado en personal asistencial de un hospital en San Luis Potosi (Argentina), de 32 individuos se obtuvieron 29 aislamientos bacterianos y una frecuencia del $52 \%$ de microorganismos potencialmente infecciosos como enterobacterias y Staphylococcus aureus; así mismo, Moscoso (6) en un hospital militar de Quito (Ecuador), realizó 87 aislamientos positivos en 79 de 100 individuos de diferentes áreas asistenciales y encontraron una frecuencia cercana al 30\% de microorganismos potencialmente infecciosos como: Klebsiella pneumoniae, Enterobacter spp, Staphylococcus aureus, Acinetobacter baumanni, Escherichia coli y Enterococo faecalis.

Aunado a las situaciones expuestas, se evidencia una problemática mayor como es el desconocimiento y las prácti- 
cas inadecuadas en los profesionales de la salud, relacionadas con los mecanismos de control y prevención, específicamente con la práctica de un correcto lavado de las manos, como fue observado en el estudio realizado por Vega, Janus \& Laskin (7) en 480 profesionales de la odontología, en el que más del $50 \%$ de los encuestados reprobaron una evaluación sobre la higienización de las manos. Por otro lado, Arenas y colaboradores (8), en un estudio multicéntrico realizado en nueve unidades de diálisis en España, encontraron que el cumplimiento de la higiene de las manos fue solo del $13,8 \%$ antes del contacto con el paciente y del 35,6\% después del contacto con el paciente.

Otra situación adicional y de especial interés está representada en las formas en cómo se han evaluado los conocimientos y las prácticas en el lavado de las manos para los profesionales de la odontología, en tal sentido Vega y colaboradores (7) aplicaron un cuestionario que incluyó solo cinco ítems de evaluación, en los cuales no se realizó ningún tipo de verificación de sus propiedades psicométricas, sumado a que podrían resultar insuficientes para demostrar la validez de constructo a través de la eliminación de ítems no explicativos de los dos dominios abordados; Myers y Naik con sus respectivos colaboradores $(9,10)$ aplicaron encuestas CAP (conocimientos, actitudes y prácticas) que incluían una cantidad considerable de ítems relacionados a estos dominios, sin embargo, de igual forma omiten los análisis propios de verificación de las propiedades psicométricas para la validación de escalas.
En el sentido de lo anteriormente expuesto y la evidencia de diversas fuentes de la literatura científica (11-14) entre muchas otras, la OMS ha recomendado la implementación en los centros de atención en salud de la estrategia multimodal para la mejora de la higiene de las manos; de la cual se han desglosado cinco herramientas para su implementación efectiva: i) cambio del sistema, ii) formación, iii) evaluación, iv) recordatorios en el lugar de trabajo y v) clima institucional de seguridad; todas ellas en pro de la viabilidad y la mejora de las prácticas de higiene de las manos, infraestructura, conocimientos y percepciones de los profesionales de la salud.

Como componentes importantes circunscritos a la estrategia multimodal: la formación y la evaluación suponen el aprendizaje continuo sobre los momentos y las técnicas correctas para la higiene de las manos y la identificación de personal y áreas, donde se enfoquen los esfuerzos y las intervenciones de los planes de acción para la implementación de dicha estrategia, es por ello que el presente estudio tiene por objetivo validar y aplicar una escala de conocimientos y prácticas en higienización de las manos antes y después de la implementación de una estrategia educativa en estudiantes de odontología de una universidad del municipio de Envigado (Antioquia, Colombia) durante el 2016.

\section{Materiales y métodos}

\section{Tipo de estudio}

Estudio longitudinal de construcción, 
conocimientos y prácticas sobre higienización de las manos.

\section{Población y sujetos}

39 estudiantes de sexto semestre de Odontología, a quienes se les realizó una intervención educativa sobre el lavado de manos, previa aplicación de la escala y posteriormente a la primera semana y seis meses después de la intervención.

\section{Criterios de inclusión}

Estudiantes de ambos sexos pertenecientes al sexto semestre del pregrado de odontología con matricula vigente.

\section{Criterios de exclusión}

Estudiantes que rechazaron su participación en el estudio, quienes al momento de la encuesta no se presentaron, exigieran remuneración o se retirasen durante el periodo de la investigación.

\section{Recolección de la información}

La fuente de información fue primaria y consistió en una encuesta con cuatro componentes: información demográfica, conocimientos y prácticas cada uno compuesto de nueve y doce ítems respectivamente con evaluación en escala tipo Likert de cinco niveles en siete ítems relacionados con los conocimientos y once ítems relacionados a las prácticas, los dos ítems restantes relacionados a las prácticas fueron observacionales, el ítem restante para el dominio conocimiento fue abierto con una observación

290 categorizada en tres niveles y además se incluyó un ítem relacionado con la me- dición del tiempo de higienización de las manos (independiente).

Los ítems surgieron a partir de la revisión del documento publicado por la OMS: "Una atención limpia es una atención más segura" (15) y aprobados, modificados o eliminados por un panel de expertos y un grupo focal (compuesto por tres odontólogos epidemiólogos y un microbiólogo) en términos de redacción, extensión, claridad y pertinencia, con el fin de dar mayor entendimiento al cuestionario (16). Además, se eliminaron categorías sin respuesta (ítems no seleccionados) e ítems en los que más del $95 \%$ de las veces se seleccionaban en la misma dirección, es decir, el ítem no era útil porque no aportaba variabilidad y por ello podía obviarse $(17,18)$. Con los ítems de cada dominio se hizo una sumatoria de las respuestas y se calcularon los puntajes con la fórmula: (Sumatoria total de la escala - puntaje mínimo) / rango) $\times 100$.

Se realizó una prueba piloto en ocho estudiantes, con el fin de evaluar la versión definitiva y el desempeño de los encuestadores, quienes fueron capacitados en asuntos relacionados con el contenido del instrumento, los aspectos éticos y las condiciones que debía reunir el lugar donde se realizó la encuesta de forma individual, asistida y anónima. La encuesta de conocimientos y prácticas fue aplicada antes de la implementación de la estrategia educativa, y una semana y seis meses después de la actividad.

La estrategia educativa tuvo en cuenta un estilo de aprendizaje reflexivo por 
parte de los estudiantes $(19,20)$, mediante la presentación de un personaje pintoresco en diferentes puntos de la facultad, el cual enfatizó los diferentes momentos y tiempos del lavado de manos en forma creativa, a través de la utilización de material didáctico para la identificación de los elementos asociados a las IRAS (pelotas de diferentes colores para la representación de los agentes microbianos), seguida de una actividad de pregunta-respuesta con premiación a las respuestas acertadas, logrando de este modo que la actividad en su conjunto fuese percibida como "divertida" por parte de los estudiantes involucrados; adicionalmente, se suministraron kits de higienización y fichas educativas con la información relacionada y se diseñaron plegables informativos que fueron ubicados en los lugares con mayor riesgo de contaminación como: el ingreso al ascensor, a los baños, a la entrada a las clínicas y en la zona de alimentación de la facultad.

\section{Análisis estadístico}

Se realizó un análisis univariado para la descripción de las características demográficas de la población de estudio y de los puntajes en los dominios de conocimientos y prácticas, a través del cálculo de frecuencias relativas y absolutas o medidas de resumen de acuerdo a la naturaleza de la variable. Para los puntajes de conocimientos y prácticas, se calcularon además el efecto piso o proporción de personas con el puntaje más bajo y el efecto techo o proporción de personas con el puntaje más alto.

\section{Validez de constructo y contenido}

Se verificó que los ítems de conocimientos y prácticas representasen adecuadamente la medición de estos para obtener inferencias válidas en un rango amplio de situaciones (16-18), para ello se realizó un análisis factorial exploratorio de componentes principales con rotación de varimax, previa verificación de su pertinencia mediante las pruebas de Kaiser-Meyer Olkin y esfericidad de Bartlett, a partir de allí se estimaron los $\%$ de variabilidad de los dominios (conocimientos y prácticas), a razón de cada uno de los ítems incluidos y las cargas factoriales (coeficientes Lambda para la relación de cada ítem de la escala con su dominio), definiéndose el porcentaje de éxito a partir del cociente (cargas factoriales $\geq 0,4$ en el dominio / total de coeficientes Lambda del Dominio) $\times 100$.

\section{Fiabilidad}

Se evaluó mediante el cálculo de alfa de Cronbach, tomando como criterio de aceptabilidad un valor $\geq 0,7$.

\section{Consistencia interna y validez discriminante}

Se calcularon coeficientes de correlación de Pearson entre los ítems y los dominios de la escala. La consistencia interna se evaluó al calcular los coeficientes de correlación de Pearson entre cada ítem y el dominio al cual pertenece, $y$ al definir el porcentaje de éxito mediante la fórmula: \% Éxito de consistencia interna $=($ Correlaciones pregunta - dimensión a la cual pertenece $\geq 0,4$ / Total de corre- 
laciones pregunta - dimensión a la cual pertenece) $\times 100$.

Para la validez discriminante se calcularon los coeficientes de correlación de Pearson entre los ítems y los dominios a los cuales no pertenecen. En esta el porcentaje de éxito para cada dominio se estableció a partir de la fórmula: \% Éxito de validez discriminante $=$ correlaciones del punto de dominio al cual no pertenece, menores que las correlaciones punto - dominio al cual pertenece / Número total de correlaciones del punto - dominio al cual no pertenece) $\times 100$.

La comparación de los puntajes en las dimensiones de conocimientos y prácticas, según los tiempos de la estrategia educativa, se realizó mediante las prueba de Friedman, dado el incumplimiento del supuesto de normalidad evaluado a través del estadístico de Shapiro Wilk y el análisis de comparaciones múltiples a través de la prueba de Wilcoxon y la corrección del nivel de significación a través de Bonferroni.

La comparación de los tiempos promedio de higienización de las manos en los tres momentos de observación se realizó mediante la prueba Anova de un factor, previa verificación de los supuestos de normalidad y homogeneidad de las varianzas evaluadas a través de los estadísticos de Shapiro Wilk y Levene respectivamente, el análisis pos hoc se realizó mediante la prueba de Bonferroni.

En todos los análisis se tomó un nivel de significación estadística menor a 0,05, 292 realizados mediante el paquete IBM SPSS $^{\circledast} 23$.

\section{Aspectos éticos}

El proyecto fue diseñado y ejecutado bajo los principios de la declaración de Helsinki de la asociación médica mundial, en el que prevaleció el respeto por la integridad e intimidad del individuo, la protección de los derechos y la garantía de la veracidad en los resultados, además de la evaluación de riesgos y beneficios. De igual modo estuvo regida por la Resolución 8430 en la que se establecen las normas científicas, técnicas y administrativas para la investigación en salud en Colombia.

\section{Resultados}

De un total de 39 estudiantes incluidos inicialmente en el estudio, 37 lo completaron, el promedio de edad entre dicha población fue $21,8 \pm 1,8$ años, con una mayor frecuencia de estudiantes del sexo femenino $(82,1 \%)$.

El análisis de consistencia interna para el dominio de conocimientos evidenció correlaciones moderadas para todos los ítems, excepto para los relacionados con "el lavado de las manos con agua y jabón" y "la eficacia de higienización con alcohol antiséptico", dichas correlaciones fueron leves pero significativas, permitiendo estimar un $\%$ de éxito de consistencia interna $=$ $77,7 \%$, a pesar de ello, estas correlaciones permitieron asociar el puntaje total del dominio con la calificación de la respuesta para estos ítems. En el análisis de la validez discriminante se encontró que solo el ítem relacionado con riesgo de infección por uñas largas mostró una correlación negativa y leve 
con el dominio de prácticas, pero menos significativas y con menor correla- ción que la evidenciada en el dominio de conocimientos (tablas 1 y 2 ).

Tabla 1. Correlaciones y coeficientes de los ítems según su dominio

\begin{tabular}{|c|c|c|c|}
\hline \multicolumn{4}{|c|}{ Conocimientos } \\
\hline Ítem & $\begin{array}{l}\text { Ítem - } \\
\text { dominio }\end{array}$ & $\begin{array}{l}\text { Ítem -otro } \\
\text { dominio }\end{array}$ & $\begin{array}{l}\text { Coeficiente } \\
\text { Lambda }\end{array}$ \\
\hline $\begin{array}{l}\text { La mejor opción para el lavado de manos es } \\
\text { el agua y el jabón }\end{array}$ & $0,290^{* *}$ & $-0,051$ & 0,647 \\
\hline $\begin{array}{l}\text { La higienización de manos con antibacterial } \\
\text { o alcohol glicerinado es una buena opción }\end{array}$ & $0,501^{* *}$ & $-0,056$ & 0,878 \\
\hline $\begin{array}{l}\text { Las uñas largas aumentan el riesgo de } \\
\text { trasmisión de infecciones }\end{array}$ & $0,436^{* *}$ & $-0,226^{*}$ & 0,493 \\
\hline $\begin{array}{l}\text { Es más eficaz el uso de alcohol antiséptico } \\
\text { que el lavado con agua y con jabón }\end{array}$ & $0,325^{* *}$ & 0,138 & 0,815 \\
\hline $\begin{array}{l}\text { El lavado de las manos antes del contacto } \\
\text { con el paciente previene la transmisión de } \\
\text { microbios desde el área de asistencia no } \\
\text { clínica a través de las manos, para proteger } \\
\text { al paciente de la colonización y de las } \\
\text { infecciones exógenas }\end{array}$ & $0,556^{* *}$ & 0,162 & 0,737 \\
\hline $\begin{array}{l}\text { El lavado de las manos inmediatamente } \\
\text { antes de acceder a un punto crítico con } \\
\text { riesgo de infección para el paciente es } \\
\text { para impedir la transmisión de microbios al } \\
\text { paciente y de un punto del cuerpo a otro del } \\
\text { mismo paciente por inoculación }\end{array}$ & $0,671^{* *}$ & 0,160 & 0,590 \\
\hline $\begin{array}{l}\text { La duración del lavado de manos clínico } \\
\text { debe ser de } 20 \text { a } 30 \text { segundos }\end{array}$ & $0,557^{* *}$ & $-0,154$ & 0,880 \\
\hline $\begin{array}{l}\text { ¿Conoce los cinco momentos de lavado } \\
\text { recomendados por la Organización Mundial } \\
\text { de la Salud? }\end{array}$ & $0,493^{* *}$ & $-0,119$ & 0,679 \\
\hline ¿Cuáles son los cinco momentos de lavado? & $0,533^{* *}$ & 0,204 & 0,819 \\
\hline Prácticas & $\begin{array}{l}\text { Ítem - } \\
\text { dominio }\end{array}$ & $\begin{array}{l}\text { Ítem - otro } \\
\text { dominio }\end{array}$ & $\begin{array}{l}\text { Coeficiente } \\
\text { Lambda }\end{array}$ \\
\hline $\begin{array}{l}\text { ¿Usted se lava las manos cuando ingresa al } \\
\text { cubículo? }\end{array}$ & $0,297^{* *}$ & 0,091 & 0,674 \\
\hline $\begin{array}{l}\text { ¿Usted se lava las manos antes de atender a } \\
\text { cada paciente? }\end{array}$ & $0,348^{* *}$ & $-0,037$ & 0,916 \\
\hline $\begin{array}{l}\text { ¿Usted se lava manos entre paciente y } \\
\text { paciente? }\end{array}$ & $0,417^{* *}$ & $-0,052$ & 0,748 \\
\hline $\begin{array}{l}\text { ¿Usted se lava las manos entre cada cambio } \\
\text { de guantes, durante la consulta? }\end{array}$ & $0,533^{* *}$ & 0,139 & 0,725 \\
\hline $\begin{array}{l}\text { ¿Usted se lava las manos antes de manejar } \\
\text { material estéril? }\end{array}$ & $0,427^{* *}$ & 0,130 & 0,871 \\
\hline
\end{tabular}


Continuación Tabla 1. Correlaciones y coeficientes de los ítems según su dominio

\begin{tabular}{|c|c|c|c|}
\hline Prácticas & $\begin{array}{l}\text { Ítem - } \\
\text { dominio }\end{array}$ & $\begin{array}{l}\text { Ítem - otro } \\
\text { dominio }\end{array}$ & $\begin{array}{l}\text { Coeficiente } \\
\text { Lambda }\end{array}$ \\
\hline $\begin{array}{l}\text { ¿Usted se lava las manos antes de salir del } \\
\text { cubículo? }\end{array}$ & $0,468^{* *}$ & $-0,042$ & 0,901 \\
\hline $\begin{array}{l}\text { ¿Usted se lava las manos después de manejar } \\
\text { material fuera del cubículo, como historia } \\
\text { clínica, celular? }\end{array}$ & $0,485^{* *}$ & 0,051 & 0,613 \\
\hline $\begin{array}{l}\text { ¿Usted se quita los anillos o pulseras para } \\
\text { lavar sus manos y atender al paciente? }\end{array}$ & $0,288^{* *}$ & $-0,194^{*}$ & 0,710 \\
\hline $\begin{array}{l}\text { ¿Usted se lava las manos después finalizar la } \\
\text { jornada de trabajo clínico? }\end{array}$ & $0,402^{* *}$ & $-0,073$ & 0,910 \\
\hline $\begin{array}{l}\text { ¿Recibe asesoría sobre el lavado de manos } \\
\text { por parte de sus docentes? }\end{array}$ & $0,433^{* *}$ & $-0,071$ & 0,568 \\
\hline $\begin{array}{l}\text { ¿Sus pacientes se lavan las manos al salir de } \\
\text { la clínica? }\end{array}$ & $0,536^{* *}$ & $-0,032$ & 0,625 \\
\hline Forma de lavado de las manos & $0,391^{* *}$ & $0,271^{* *}$ & 0,623 \\
\hline
\end{tabular}

* El coeficiente de correlación de Pearson es significativo en 0,05.

**El coeficiente de correlación de Pearson es significativo en 0,01.

Fuente: elaboración propia.

Tabla 2. Descripción y evaluación de la escala

\begin{tabular}{lcc}
\hline & Conocimientos & Prácticas \\
\hline Media \pm DE & $72,2 \pm 10$ & $66,6 \pm 7,7$ \\
Mediana (rango intercuartil) & $70,9(64,5-80,6)$ & $66,6(62,5-70,8)$ \\
Rango (mín -máx) & $54,9-96,7$ & $39,6-79,2$ \\
Efecto piso \% & 2,6 & 2,6 \\
Efecto techo \% & 2,6 & 10,3 \\
\% éxito consistencia interna & 77,7 & 72,7 \\
\% éxito validez discriminante & 100 & 100 \\
\% éxito constructo & 100 & 100 \\
Fiabilidad ( $\alpha$ de Cronbach) & 0,71 & 0,73 \\
\hline
\end{tabular}


Los análisis de validez de constructo y contenido para dicho dominio evidenciaron la extracción de cuatro ítems que explicaban el $72,4 \%$ de la varianza en los conocimientos, sin embargo, las cargas factoriales estimadas para los nueve ítems incluidos cumplían con el criterio de aceptación para el éxito de la validación contenido-constructo; por otro lado, para el análisis de correlación entre los ítems del dominio se estimó una $\alpha$ de Cronbach $=0,71$, lo que significa una confiabilidad aceptable para esta dimensión (tablas 1 y 2).

En el dominio de prácticas, cuatro ítems presentaron correlaciones leves pero significativas, representando un $\%$ de éxito de consistencia interna $=72,7 \%$; el análisis de validez discriminante evidenció que solo el ítem "retiro de accesorios para el lavado de las manos y atención al paciente" presentó una correlación significativa pero menor a la calculada para la asociación ítem-dominio, al permitir estimar un éxito del $100 \%$ para la capacidad discriminante de los ítems relacionados con las prácticas (tablas 1 y 2 ).

La validez de constructo y de contenido del dominio de prácticas fue satisfactoria dado el porcentaje de éxito obtenido a partir de la estimación de las cargas factoriales para los 12 ítems y cuyos valores estuvieron por encima de $0,4 \mathrm{e}$ identificaron cinco componentes con capacidad de explicar el $71,4 \%$ de la varianza del dominio. La confiabilidad de dicho dominio también resultó aceptable con una estimación del a de Cronbach $=0,71$ (tablas 1 y 2 ).
En términos generales, la escala evidenció un comportamiento satisfactorio para las estimaciones de los efectos piso y techo con bajas proporciones de individuos, presentando el menor y mayor puntaje respectivamente, los puntajes promedio obtenidos al momento de la validación de la escala y previo a la implementación de la estrategia educativa fueron $72,2 \pm 10$ y $66,6 \pm 7,7$ en conocimientos y prácticas respectivamente (tabla 2).

La evaluación de la efectividad de la intervención educativa implementada mediante la observación de los tiempos del lavado de las manos y los puntajes en conocimientos y prácticas entre los estudiantes, evidenció que los promedios de tiempo de lavado fueron significativamente mayores después de la implementación (figura 1), así mismo para el dominio de conocimientos fueron significativamente mayores los puntajes obtenidos la semana después de la intervención ( $\mathrm{Me}=87$ puntos), comparados con las puntuaciones antes de la intervención $(\mathrm{Me}=71, \mathrm{Vp}<$ $0,001)$ y seis meses después $(81=$ puntos, $\mathrm{Vp}=0,007)$; la comparación entre estos dos últimos también evidenció diferencias estadísticamente significativas $(\mathrm{Vp}=0,011)$; para el dominio de prácticas, las diferencias se encontraron en la comparación de los puntajes antes de la intervención ( $\mathrm{Vp}=0,033)$, (Me $=67$ puntos) y la semana después ( $\mathrm{Me}=71$ puntos); los puntajes comparados entre los dos momentos después de la intervención no mostraron diferencias significativas $(\mathrm{Vp}=1)$ (figuras 2 y 3$)$. 


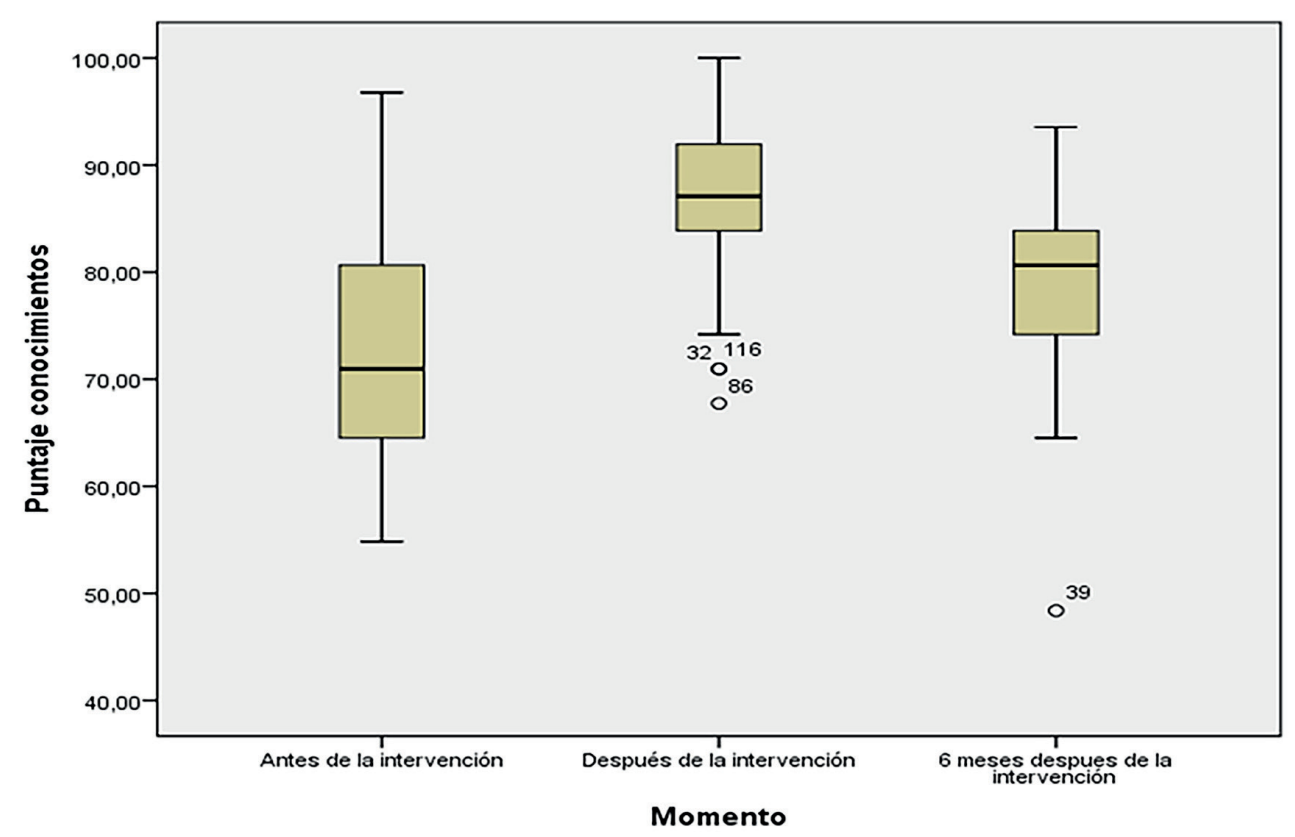

Figura 1. Tiempos promedio de desinfección en los tres momentos de la intervención Fuente: elaboración propia.

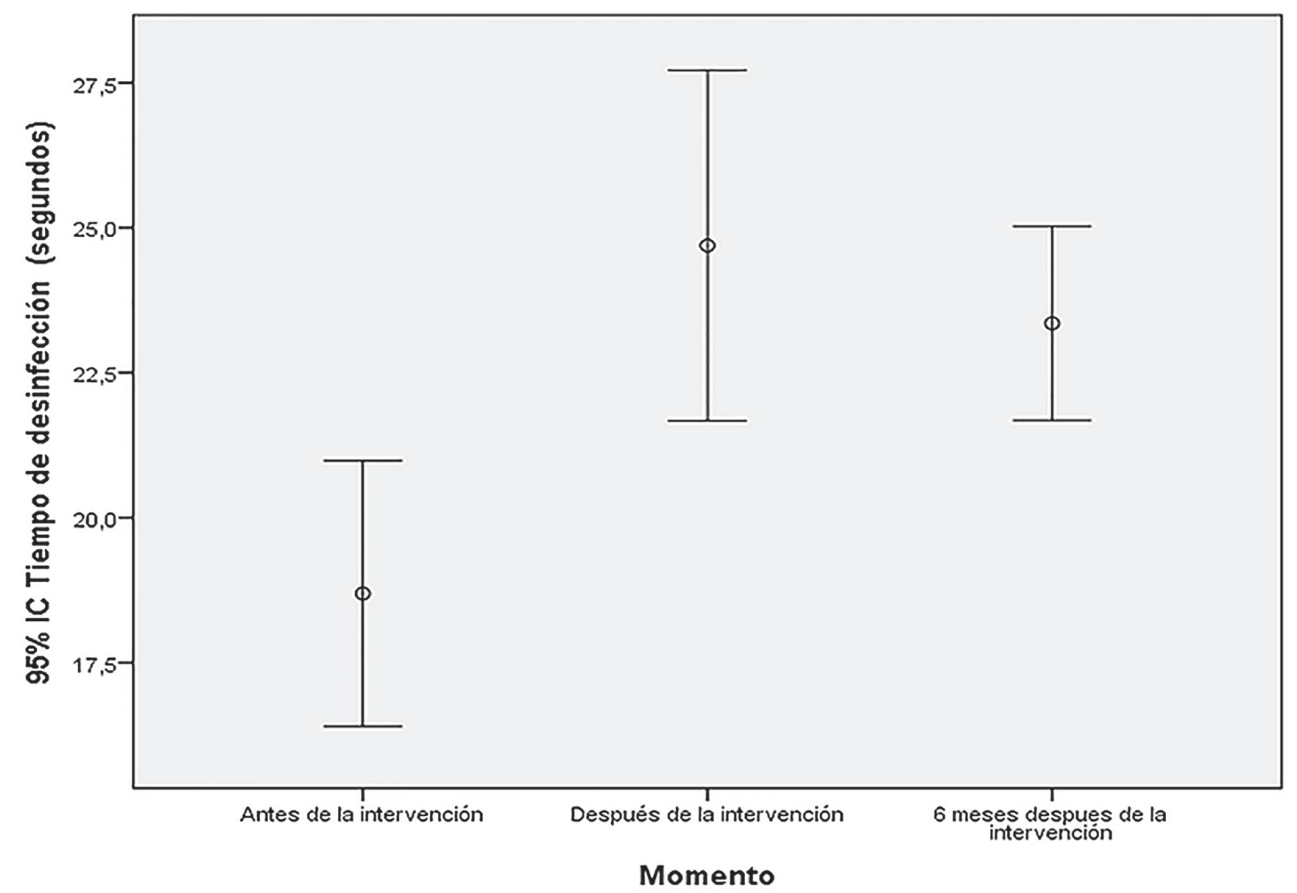

296 Figura 2. Puntajes en el dominio de conocimientos en los tres momentos de la intervención Fuente: elaboración propia. 


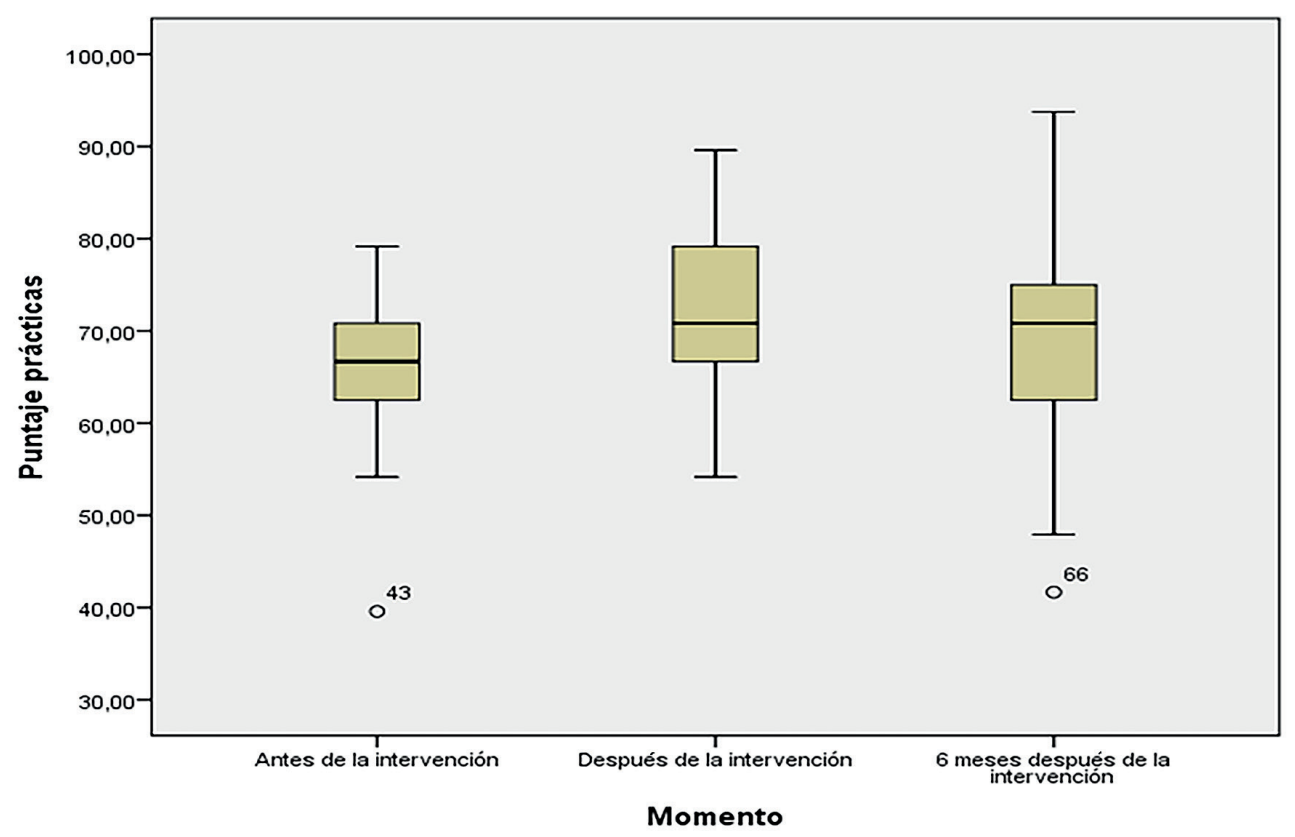

Figura 3. Puntajes en el dominio de prácticas en los tres momentos de la intervención Fuente: elaboración propia.

\section{Discusión}

La validación de la escala sobre conocimientos y prácticas en el lavado de las manos presentó una fiabilidad y validez aceptables; su aplicación como herramienta de evaluación de la estrategia educativa constituyó un elemento de utilidad, dada la mejoría significativa en los puntajes de dichos dominios y evidencia la pertinencia de su aplicación dentro del marco de estrategia multimodal definido por la OMS.

El estudio de las escalas de conocimientos y prácticas sobre la higienización de las manos como un modelo teórico permite la orientación de medidas preventivas que disminuyan el riesgo de adquisición de IRAS, al tiempo que supone su emergencia como un tema de investigación dentro de los incluidos por la OMS en su alianza mundial por la seguridad del paciente, dado el impacto de las infecciones asociadas a la atención sanitaria y el llamado de esta organización para el aumento del desarrollo metodológico y de instrumentos adecuados que fortalezcan la capacidad de investigación, especialmente en entornos sanitarios de países en desarrollo (21).

El mejoramiento en el puntaje de las prácticas de higienización de las manos después de la implementación de la estrategia educativa representó un desenlace similar frente al resultado obtenido en el estudio de Creedo (22) en personal asistencial de un centro hospitalario en Irlanda; quien a través de la aplicación de una estrategia educativa multifacética fundamentada en la predisposición 
de los trabajadores hacia la adopción de buenos hábitos de higiene (recordatorios en lugares de trabajo y áreas de circulación), el reforzamiento (realimentación individual sobre la evaluación previa a la intervención) y la promoción y habilitación del uso de desinfectantes con alcohol en las áreas de trabajo, logró un significativo aumento en las frecuencias de cumplimiento de las guías de higienización establecidas. De forma similar, Pittet, Arntz y sus colaboradores $(23,24)$ lograron respectivamente en hospitales de Genova (Suiza) y Nijmegen (Holanda) aumentar la proporción de cumplimiento de las guías de higienización después de la aplicación de estrategias educativas multimodales, al mismo tiempo Pittet y colaboradores observaron una disminución en las frecuencias de infecciones nosocomiales y trasmisión de Staphylococcus aureus meticilino resistente en el lugar de estudio.

Respecto a lo observado en el dominio de conocimientos, los resultados del presente estudio convergen con los obtenidos por Sánchez y colaboradores (25) en 228 trabajadores sanitarios de un hospital universitario de la ciudad de Alicante, cuya metodología basada en sesiones teóricas de educación y provisionamiento de soluciones desinfectantes a los empleados, lograron una disminución en los porcentajes de respuestas inadecuadas en un test sobre la higienización de las manos y un aumento en las frecuencias de cumplimiento a las recomendaciones impartidas; paralelamente durante uno de los periodos de estudio (2005-2006) observaron una disminución de la frecuencia de infecciones no- socomiales respecto al año previo a la intervención educativa (2004).

El aumento observado en los tiempos promedio de la desinfección de manos constituyó una situación de adherencia por parte de los estudiantes a las prácticas impartidas desde la intervención educativa y, a su vez, concordantes con la recomendación de la OMS de un tiempo mínimo entre 20 y 30 segundos (cuando las manos no están visiblemente sucias) para la reducción de la microbiota colonizante hasta en un factor de $10^{3}$ unidades, especialmente aquella transitoria potencialmente infecciosa como: Escherichia coli, Klebsiella spp, Pseudomonas aeruginosa, Staphylococcus aureus y algunos virus cuando el lavado de las manos es adecuado en términos de tiempo, con una solución desinfectante y técnica (26).

La evidencia expuesta, agregada a lo desarrollado en el presente estudio, manifiesta la aplicación de las diversas herramientas de la estrategia multimodal propuesta por la OMS: formación, evaluación, retroalimentación y recordatorios en los lugares; cuyos resultados han impactado favorablemente los conocimientos y las prácticas en los trabajadores del área de la salud sobre la higienización de las manos, con un efecto colateral benéfico para la salud pública como es la disminución de las infecciones intrahospitalarias y lo que implícitamente supone en términos de costo-beneficio para el sistema de salud.

Los profesionales de la odontología, cuyo ejercicio implica la realización frecuente de diversas intervenciones inva- 
sivas en los pacientes, para las cuales su recuperación en la mayoría de los casos es de carácter ambulatorio, requieren desde su proceso formativo la adopción de estas sencillas prácticas de higienización impartidas desde lo teórico y una continua retroalimentación y monitorización; constituidas en actividades encaminadas a la disminución del riesgo de IRAS. Al mismo tiempo se hace fundamental un seguimiento al individuo intervenido en un periodo crítico, con el fin de obtener registros que conlleven a la cuantificación de estas condiciones tan pobremente identificadas en el entorno ambulatorio.

Como limitaciones del presente trabajo se señala la imposibilidad de contar con un sistema categorización de los puntajes en la escala que permitiese calificar los puntos en los conocimientos y prácticas en el inicio del estudio y después de la intervención educativa, así mismo, el bajo número de individuos que constituyeron la población de estudio por efectos de disponibilidad y la intencionalidad de este (semestre en el que se inician las prácticas clínicas), lo cual pudo suponer problemas de potencia estadística en la verificación de la escala y en ese sentido explicar la baja correlación entre algunos ítems y sus dominios al no contener un rango más amplio de valores distribuidos dentro de la puntuación de la escala, a pesar de ello se dejan incluidos en la escala, para efectos de futuras validaciones en un número más amplio de individuos; además, no se complementó la evaluación del instrumento con otras propiedades psicométricas importantes como la confiabilidad intra e inter observador, la sensibilidad al cambio y la validez de criterio y validez convergente/ divergente; a pesar de lo anterior, la validación lograda representa una herramienta importante para investigaciones futuras que busquen evaluar la escala expuesta en diferentes disciplinas del sector asistencial en salud.

\section{Conclusión}

Los resultados permiten concluir que la escala construida y evaluada tuvo un buen desempeño psicométrico en la población de estudiantes universitarios, además de ser sensible frente a la aplicación de una intervención educativa, lo que supone su utilidad en el monitoreo de este tipo de procesos que permitan estimar su eficacia.

La buena confiabilidad y la validez en la escala construida representan la disponibilidad de un instrumento para la evaluación de los conocimientos y las prácticas en higienización de las manos en profesionales de la salud y la orientación de procesos de información, comunicación y educación. La intervención educativa evidenció un mejoramiento en las prácticas y en los conocimientos de higienización de lavado de manos, sin embargo, se requiere un continuo refuerzo y seguimiento para garantizar el desarrollo de una práctica clínica más segura. 


\section{Referencias bibliográficas}

1.Organización Mundial de la Salud. La higiene de las manos en la asistencia ambulatoria y domiciliaria y en los cuidados de larga duración: guía de aplicación de la estrategia multimodal de la OMS para la mejora de la higiene de las manos y del modelo "Los cinco momentos para la higiene de las manos [Internet]. 2013 [citado 2018 abr. 03]. Disponible en: http://apps.who.int/iris/bitstream/ handle/10665/84918/9789243503370_ spa.pdf?sequence $=1$

2. Willy M. The epidemiology and control of communicable diseases (in the outpatient setting). Lippincott's Primary Care Practice. 1999;3(1):82-92.

3. Aranaz JM, Aibar C, Limón R, Mira JJ, Vitaller J, Agra Y, et al. A study of the prevalence of adverse events in primary healthcare in Spain. European Journal of Public Health. 2012;22(1):921-5. Doi: 10.1093/eurpub/ckr168.

4. Schulz M, Mielke M, Wischnewski N. Clusters of infectious diseases in German nursing homes: observations from a prospective infection surveillance study, October 2008 to August 2009. Euro Surveillance. 2011;16(22):18-22.

5. Aguilar V, Velarde L, Martínez A, Fragoso L. Disminución de la carga microbiana aislada de manos, del personal del área de quirófano de un hospital de segundo nivel, después del procedimiento de lavado. Enf Infec Microbiol. 2011;31(3):89-92.

6. Moscoso M. Colonización por microorganismos en las manos del personal de salud en áreas críticas del hospital de Quito $\mathrm{n}^{\circ} 1$ "policía nacional" en el periodo febrero 2016-junio 2016 [Internet]. Ecuador: Universidad central del Ecuador; 2016. Disponible en: http://www.dspace. uce.edu.ec/handle/25000/9242
7. Vega G, Janus C, Laskin D. Hand-washing knowledge and practices among dentists and dental specialists. Quintessence International. 2012;43(5):429-34.

8. Arenas MD, Sánchez-Payá J, Barril G, García-Valdecasas J, Gorriz JL, Soriano $A$, et al. A multicentric survey of the practice of hand hygiene in haemodialysis units: factors affecting compliance. Nephrology, Dialysis, Transplantation. 2005;20:1164-71.

9. Myers R, Larson E, Cheng B, Schwartz A, Da Silva K, Kunzel C. Hand hygiene among general practice dentists: A survey of knowledge, attitudes and practices. J Am Dent Assoc. 2008;139:948-57.

10. Naik S, Khanagar S, Kumar A, Vadavadagi S, Neelakantappa H. Ramachandra Knowledge, attitude, and practice of hand hygiene among dentists practicing in Bangalore city - A cross-sectional survey [Internet]. J Int Soc Prev Community Dent. 2014 [citado 2018 May. 10];4(3):159-63. Doi: 10.4103/2231-0762.142013.

11. Pittet D, Allegranzi B, Sax H, Dharan S, Pessoa-Silva CL, Donaldson L, et al. Evidence-based model for hand transmission during patient care and the role of improved practices. Lancet Infect Diseases, 2006;6(10):641-52.

12. Cohen HA, Kitai E, Levy I, Ben-Amitai D.Handwashing patterns in two dermatology clinics. Dermatology. 2002;205:358-61.

13. Pan A, Domenighini $F$, Signorini $L$, Assini R, Catenazzi P, Lorenzotti S, et al. Adherence to hand hygiene in an Italian long term care facility. American Journal of Infection Control, 2008; 36:495-497. 
14. Smith A, Carusone SC, Loeb M. Hand hygiene practices of health care workers in long-term care facilities. American Journal of Infection Control. 2008;36:492-4.

15. Organización Mundial de la Salud. Una atención limpia es una atención más segura. [fecha desconocida] [citado 2017 sept. 11]. Disponible en: http://www.who. int/gpsc/es/

16. Arribas M. Diseño y validación de cuestionarios. Matronas Profesión, 2004;5(17):23-29.

17. Lamprea JA, Gómez C. Validez en la validación de escalas. Rev. Colomb. Psiquiat. 2007;36(2):340-8.

18. Sánchez R, Echeverry J. Validación de escalas de medición en salud. Rev Salud pública. 2004;6(3):302-18.

19. Alonso C, Gallego D, Honey P. Los estilos de aprendizaje: procedimientos de diagnóstico y mejora. 3a ed. Bilbao: Ediciones Mensajero; 2007. 221 p.

20. Yancen L, Consuegra D, Herrera K, Pacheco B, Díaz D. Estrategias educativas utilizadas por los docentes del programa de Enfermería de una universidad de la ciudad de Barranquilla (Colombia) frente a los estilos de aprendizaje de los estudiantes de este programa. Rev Salud Uninorte, 2013;29(3):405-16.

21. OMS. Alianza Mundial para la seguridad del paciente: Reto mundial en pro de la seguridad del paciente. 20052006 [Internet]. 2005 [citado 2018 May 23]. Disponible en: http://www.who. int/patientsafety/information_centre/ GPSC_Launch_sp.pdf

22. Creedon SA. Healthcare workers' hand decontamination practices: compliance with recommended guidelines. J Adv Nurs. 2005;51:208-16.

23. Pittet $D$, Hugonnet $S$, Harbarth $S$, Mourouga P, Sauvan V, Touveneau S, et al. Effectiveness of a hospital-wide programme to improve compliance with hand hygiene. Infection Control Programme. Lancet. 2000;356:1307-12.

24. Arntz PR, Hopman J, Nillesen M, Yalcin E, Bleeker-Rovers CP, Voss A. Effectiveness of a multimodal hand hygiene improvement strategy in the emergency department. American Journal of Infection Control. 2016;44:1203-7.

25. Sánchez J, Fuster M, García C, Gracia R, García P, San Juan A. et al. Evaluación de un programa de actualización de las recomendaciones sobre la higiene de manos. Anales Sis San Navarra. 2007;30(3):343-52.

26. Kampf G, Kramer A. Epidemiologic background of hand hygiene and evaluation of the most important agents for scrubs and rubs. Clin Microbiol Rev. 2004;17(4):863-93. Doi: 10.1128/ CMR.17.4.863-893.2004 


\section{Anexo 1. Encuesta de conocimientos y prácticas sobre la higienización de las manos}

Fecha: Código n.

Responsable:

Edad del participante:

Sexo: M F

Realice la higienización (observación del investigador encuestador)

Tiempo:

\begin{tabular}{|c|l|c|c|c|}
\hline Pasos & & Sí & No & \\
\hline 1 & Frota las palmas & & & \multirow{2}{*}{ a Realiza todos los pasos } \\
\hline 2 & Frota los dorsos & & & \multirow{2}{*}{ c Noaliza algunos__ealiza ninguno_ } \\
\hline 3 & Entrelaza los dedos & & & \\
\hline 4 & Frota dedo pulgar & & & \\
\hline 5 & Frota puntas de los dedos & & & \\
\hline
\end{tabular}

En las siguientes preguntas conteste con una X en la casilla que usted considere:

\begin{tabular}{|l|l|l|l|l|l|}
\hline \multicolumn{1}{|c|}{ Preguntas } & $\begin{array}{c}\text { Totalmente } \\
\text { de acuerdo }\end{array}$ & De acuerdo & Indiferente & $\begin{array}{c}\text { En } \\
\text { desacuerdo }\end{array}$ & $\begin{array}{c}\text { Totalmente } \\
\text { en } \\
\text { desacuerdo }\end{array}$ \\
\hline $\begin{array}{l}\text { 1. La mejor opción } \\
\text { para el lavado de } \\
\text { manos es el agua } \\
\text { y el jabón }\end{array}$ & & & & & \\
\hline $\begin{array}{l}\text { 2. La higienización } \\
\text { de manos con } \\
\text { antibacterial } \\
\text { o alcohol } \\
\text { glicerinado es una } \\
\text { buena opción }\end{array}$ & & & & & \\
\hline
\end{tabular}




\begin{tabular}{|c|c|c|c|c|c|}
\hline Preguntas & $\begin{array}{l}\text { Totalmente } \\
\text { de acuerdo }\end{array}$ & De acuerdo & Indiferente & $\begin{array}{c}\text { En } \\
\text { desacuerdo }\end{array}$ & $\begin{array}{c}\text { Totalmente } \\
\text { en } \\
\text { desacuerdo }\end{array}$ \\
\hline $\begin{array}{l}\text { 3. Las uñas } \\
\text { largas aumentan } \\
\text { el riesgo de } \\
\text { trasmisión de } \\
\text { infecciones }\end{array}$ & & & & & \\
\hline $\begin{array}{l}\text { 4. Es más eficaz } \\
\text { el uso de alcohol } \\
\text { antiséptico que el } \\
\text { lavado con agua y } \\
\text { con jabón }\end{array}$ & & & & & \\
\hline $\begin{array}{l}\text { 5. El lavado de las } \\
\text { manos antes del } \\
\text { contacto con el } \\
\text { paciente previene } \\
\text { la transmisión de } \\
\text { microbios desde el } \\
\text { área de asistencia } \\
\text { no clínica, a través } \\
\text { de las manos, } \\
\text { para proteger al } \\
\text { paciente de la } \\
\text { colonización y de } \\
\text { las infecciones } \\
\text { exógenas }\end{array}$ & & & & & \\
\hline $\begin{array}{l}\text { 6. El lavado } \\
\text { de las manos } \\
\text { inmediatamente } \\
\text { antes de acceder } \\
\text { a un punto crítico } \\
\text { con riesgo de } \\
\text { infección para } \\
\text { el paciente es } \\
\text { para impedir } \\
\text { la transmisión } \\
\text { de microbios al } \\
\text { paciente y de un } \\
\text { punto del cuerpo } \\
\text { a otro del mismo } \\
\text { paciente por } \\
\text { inoculación }\end{array}$ & & & & & \\
\hline $\begin{array}{l}\text { 7. La duración del } \\
\text { lavado de manos } \\
\text { clínico debe ser de } \\
20 \text { a } 30 \text { segundos }\end{array}$ & & & & & \\
\hline
\end{tabular}


INVESTIGACIONES ANDINA No. 39, Vol. 21

A las siguientes preguntas conteste con una X la casilla que usted considere:

\begin{tabular}{|l|l|l|l|l|l|}
\hline \multicolumn{1}{|c|}{ Preguntas } & Nunca & Casi nunca & $\begin{array}{c}\text { Algunas } \\
\text { veces }\end{array}$ & $\begin{array}{c}\text { Casi } \\
\text { siempre }\end{array}$ & Siempre \\
\hline $\begin{array}{l}\text { 8. iUsted se } \\
\text { lava las manos } \\
\text { cuando ingresa al } \\
\text { cubículo? }\end{array}$ & & & & & \\
\hline $\begin{array}{l}\text { 9. ¿Usted se lava } \\
\text { las manos antes } \\
\text { de atender a cada } \\
\text { paciente? }\end{array}$ & & & & & \\
\hline $\begin{array}{l}\text { 10. ¿Usted se } \\
\text { lava manos } \\
\text { entre pacientey } \\
\text { paciente? }\end{array}$ & & & & & \\
\hline $\begin{array}{l}\text { 11. ¿Lava sus } \\
\text { manos entre } \\
\text { cada cambio de } \\
\text { guantes, durante } \\
\text { la consulta? }\end{array}$ & & & & & \\
\hline $\begin{array}{l}\text { 12. iUsted se } \\
\text { lava las manos } \\
\text { antes de manejar } \\
\text { material estéril? }\end{array}$ & & & & & \\
\hline $\begin{array}{l}\text { 13. ¿Usted se } \\
\text { lava las manos } \\
\text { antes de salir del } \\
\text { cubículo? }\end{array}$ & & & & & \\
\hline $\begin{array}{l}\text { 14. iUsted se lava } \\
\text { las manos después } \\
\text { de manejar } \\
\text { material fuera del } \\
\text { cubículo, como } \\
\text { historia clínica, } \\
\text { celular? }\end{array}$ & & & & & \\
\hline $\begin{array}{l}\text { 15. iSe quita } \\
\text { anillos o pulseras } \\
\text { para lavar sus } \\
\text { manosy atender } \\
\text { al paciente? }\end{array}$ & & & & & \\
\hline $\begin{array}{l}\text { 16. iUsted se lava } \\
\text { las manos después } \\
\text { finalizar la jornada } \\
\text { de trabajo clínico? }\end{array}$ & & & & & \\
\hline
\end{tabular}




\begin{tabular}{|l|l|l|l|l|l|}
\hline \multicolumn{1}{|c|}{ Preguntas } & Nunca & Casi nunca & $\begin{array}{c}\text { Algunas } \\
\text { veces }\end{array}$ & $\begin{array}{c}\text { Casi } \\
\text { siempre }\end{array}$ & Siempre \\
\hline $\begin{array}{l}\text { 17. 2Recibe } \\
\text { asesoría sobre el } \\
\text { lavado de manos } \\
\text { por parte de sus } \\
\text { docentes? }\end{array}$ & & & & & \\
\hline $\begin{array}{l}\text { 18. Sus pacientes } \\
\text { se lavan las manos } \\
\text { al salir de la } \\
\text { clínica? }\end{array}$ & & & & & \\
\hline
\end{tabular}

19. ¿Usted conoce los cinco momentos del lavado de manos recomendados por la Organización Mundial de la Salud? a. Si b. No ¿Cuáles son?:

\begin{tabular}{|c|l|l|}
\hline a. & Ninguno de los momentos & \\
\hline b. & Algunos de los momentos & \\
\hline c. & Todos los momentos & \\
\hline
\end{tabular}

